\title{
Risk adjusted momentum strategies: a comparison between constant and dynamic volatility scaling approaches
}

\author{
Minyou Fan ${ }^{\mathrm{a}}$, Youwei Li ${ }^{\mathrm{a}}$, Jiadong Liu ${ }^{\mathrm{a}, *}$ \\ ${ }^{a}$ Queen's Management School, Queen's University Belfast, UK
}

\begin{abstract}
We compare the performance of two volatility scaling methods in momentum strategies: (i) the constant volatility scaling approach of Barroso and SantaClara (2015), and (ii) the dynamic volatility scaling method of Daniel and Moskowitz (2016). We perform momentum strategies based on these two approaches in an asset pool consisting of 55 global liquid futures contracts, and further compare these results to the time series momentum and buy-andhold strategies. We find that the momentum strategy based on the constant volatility scaling method is the most efficient approach with an annual return of $15.3 \%$.
\end{abstract}

Keywords: Cross-sectional momentum, Time series momentum, Momentum crashes, Volatility scaling

\section{Introduction}

Over the past two decades, momentum has become one of the most widely studied financial market phenomenons and profitable trading rules, in both academia and industry. Momentum refers to the cross-sectional momentum (henceforth, XSMOM), where abnormal profits are generated by longing the best-performed stocks (winner) and shorting the poor-performed stocks

${ }^{*}$ Corresponding Author. Address: 185 Stranmillis Road, Belfast, UK, BT9 5EE. Email: jliu15@qub.ac.uk. 
(loser) in the past 3-12 months (Jegadeesh and Titman, 1993). However, recent studies suggest that momentum strategies, although generate persistent abnormal returns over time and across different asset classes ${ }^{1}$, suffer from occasional large crashes (i.e. momentum crash) ${ }^{2}$. To address this issue, volatility scaling methods are used to control risks of momentum strategies, see, e.g., Boguth et al. (2011), Wang and Xu (2015), Barroso and Santa-Clara (2015) and Daniel and Moskowitz (2016).

According to recent literature, there are two prevalent volatility scaling methods: (i) the Constant Volatility Scaling Approach (henceforth, CVS) documented by Barroso and Santa-Clara (2015), and (ii) the Dynamic Volatility Scaling Approach (henceforth, DVS) of Daniel and Moskowitz (2016). A CVS momentum strategy weights different instruments in the portfolio based on the ratio between a constant target volatility and realised volatility. In contrast, a DVS momentum strategy weights its instruments depending on the ratio between the expected market returns and realised volatility. Both approaches perform efficiently in U.S stock markets as seen in Barroso and Santa-Clara (2015) and Daniel and Moskowitz (2016), but it is still under debate that which one is better.

The rationales of the two approaches are qualitatively different but related to each other. Barroso and Santa-Clara (2015) argue that the main risks of momentum strategies are the systematic risks which account for $87 \%$ of total risks. Hence, they introduce the CVS to control for systematic risks. Whereas Daniel and Moskowitz (2016) suggest that the major risks of momentum strategies are the time-varying beta risks caused by investors' hedging positions. To reduce these risks, the authors develop the DVS. On the other hand, these two approaches are highly related. According to Daniel and Moskowitz (2016), the two approaches yield to the same results, when the Sharpe ratios of momentum strategies are time invariant.

\footnotetext{
${ }^{1}$ Evidence of momentum has also been found in international stock markets, see, e.g., Fama and French (1998); Teplova and Mikova (2015), emerging markets see, e.g., Rouwenhorst (1999); Zaremba and Szyszka (2016), country indices, see, e.g., Asness et al. (1997), industries, see, e.g., Moskowitz and Grinblatt (1999), size and B/M factors, see, e.g., Lewellen (2002), commodities, see, e.g., Miffre and Rallis (2007); Shen et al. (2007), and global asset classes, see, e.g., Asness et al. (2013).

${ }^{2}$ See Daniel and Moskowitz (2016).
} 
In this paper, we implement volatility scaled momentum strategies based on both approaches, i.e. CVS based XSMOM and DVS based XSMOM, in an asset pool consisting of 55 futures instruments similar to Asness et al. (2013) and Kim et al. (2016). Results confirm the existence of momentum crash in futures markets across different asset classes. Then we employ the Fama-French-Carhart four-factor model to evaluate the performance of these two scaling methods. The regression results show that the Jense's alpha of CVS based XSMOM strategy (1.93\%) is significantly higher than the alpha of DVS based XSMOM strategy (1.43\%) using our sample data from November, 1991 to May, 2017.

For a more in-depth comparison between the two aforementioned approaches, we divide the entire period into three sub-periods according to Daniel and Moskowitz (2016), who claim that the motivation of designing DVS is due to the relationship between sentiment and realised volatilities. In other words, when investors experience financial stress (e.g., the 2007-2008 global financial crisis), their market activities would increase the volatilities dramatically. Hence, we generate 3 sub-periods (1991-2006, 2006-2010 and 2010-2017) based on the 2007-2008 financial crisis. During pre sub-period, we find that the abnormal returns of CVS based XSMOM are significantly higher than the XSMOM returns based on DVS. However, the superiority of CVS becomes statistically insignificant during crisis and post crisis periods.

In our cross-strategy comparison, we include a standard buy-and-hold strategy and the time series momentum strategy (henceforth, TSMOM) of Moskowitz et al. (2012) as two benchmarks. In contrast to XSMOM which focuses on relative returns, a TSMOM signal only depends on the historical returns of each future contract on its own. In particular, a TSMOM strategy generates profits by longing (shorting) the contracts with positive (negative) returns in the past 3-12 months. Moreover, we implement a time-varying weighting scheme based on volatility scaling as in Moskowitz et al. (2012). This method not only improves the performance of TSMOM strategy, but also allows a fair comparison with our volatility adjusted XSMOM strategies. Empirical results shows that the volatility scaled benchmark strategies outperform the unscaled strategies as is also confirmed in Kim et al. (2016). However, the CVS based XSMOM is still the most profitable trading strategy among all of them. 
In summary, this paper contributes to the literature in the following manners. First, we identify the momentum crash in futures markets, and hence demonstrate the reasonableness to employ the volatility scaling approaches. Second, we find that the CVS based XSMOM is more efficient and profitable than the DVS based XSMOM with the difference being statistically significant. Finally, the expanded comparison suggests that the CVS based XSMOM strategy performs significantly better than the scaled TSMOM and buy-and-hold strategies.

The remainder of this paper is organised as follows. In Section 2, we provide the data sources and the summary statistics. Section 3 presents the ways in calculating XSMOM strategies and different volatility scaling methods. Then, we discuss the performance of different XSMOM strategies and regression results in Section 4. Finally, Section 5 concludes.

\section{Data}

Similar to Kim et al. (2016), we collect monthly prices from 55 global liquid futures instruments with updated time range (June, 1986 to May, 2017). The asset pool consists of 24 commodity contracts, 13 sovereign bond contracts, 9 currency contracts and 9 equity index contracts. In this section, both the data sources and summarised statistics of our sample data are reported.

\subsection{Data sources}

For each instrument, the continuous monthly futures prices are constructed by rolling all the nearest contracts to form a long time series from Bloomberg. In commodity sector, Aluminium, Copper, Nickel, Zinc are from London Metal Exchange (LME), The Brent Crude, Gas Oil, Cotton, Coffee, Cocoa, Sugar are collected from Intercontinental Exchange (ICE), Live Cattle, Lean Hogs are from Chicago Mercantile Exchange centre (CME), Corn, Soy beans, Soy Meal, Soy Oil and Wheat are downloaded from Chicago 
Board of Trade (CBOT), WTI crude, Unleaded Gasoline, Heating Oil, Natural Gas are from New York Commodity Exchange (COMEX). Platinum is collected from Tokyo Commodity Exchange (TOCOM). In bond sector, we include Australia 3-year and 10-year Bond, Euro 2-year, 5-year, 10-year and 30-year Bond, Canada 10-year Bond, Japan 10-year Bond, Long Gilt (UK 10-year), US 2-year, 5-year, 10-year and 30-year treasury. In currency sector, we cover the currencies of Australia, Canada, Euro, Japan, New Zealand, Norway, Sweden, Switzerland, UK against US dollar. While the universe of equity sector consists of stock indices futures from SPI 200 (Australia), CAC 40 (France), DAX (Germany), FTSE/MIB (Italy), TOPIX (Japan), AEX (Netherlands), IBEX 35 (Spain), FTSE 100 (UK), and S\&P 500 (U.S).

In order to explore the properties of different asset classes, we collect the monthly returns of four major financial asset class indices including MSCI world Index, S\&P GSCI, Barclays Aggregate Bond Index and the US Dollar Index. These factors are downloaded from Bloomberg. Besides, we also include the percentage changes of Fama-French factors in the regression analysis. They are Fama and French (1993) small market capitalization minus big $(s m b)$, high book-to-market ratio minus low $(\mathrm{hml})$, and Carhart (1997) premium on winner minus loser (umd). The above data is downloaded from K. French's website.

\subsection{Summarized statistics}

In Table 1, we summarise the descriptive statistics of the original series. The Bloomberg tickers, sectors, date of the first available data for each series, annualised arithmetic means and standard deviations are presented. Most futures have positive long term annualised means, while some of the currencies and index futures show slightly negative returns. Regarding volatility, we find that it varies across different asset classes.

In sector level, the government bond shows the lowest average standard deviation $(6.72 \%)$, but it does not generate the lowest return. The average return cross different contracts in currency sector is only $0.72 \%$, which is the smallest among all the four sectors. In contrast, The volatility and return

of commodities and equities are much higher than those of currencies and 
bonds. Specifically, the FTSE/MIB index contracts provide the lowest annualised return (-1.33\%) among all contracts. The EURO 2-years bond contracts exhibit the lowest annualised stander deviation (1.33\%). The Natural Gas contracts display the highest annualised return and stander deviation of $16.56 \%$ and $72.53 \%$, respectively.

\section{Methodology}

This section presents details of methodologies used in this study. We first explain our method in calculating the XSMOM returns. Then, we focus on the volatility estimation and introduce the two volatility scaling approach, CVS and DVS. Finally, the two benchmarks, buy-and-hold and TSMOM, are specified in Section 3.3.

\subsection{Cross-section momentum strategies}

The XSMOM strategy is constructed by longing the winners and shorting the losers over a certain look-back period. According to Kim et al. (2016), we select the look-back period of 6 months and holding period of 1 month. We divide the entire asset pool into deciles, where we buy the top-performed decile and short the bottom one. To make sure that there are enough futures contracts to be included in our momentum portfolio, we implement our momentum strategies when there are at least 45 contracts available in the dataset, so that the number of instruments in the top/bottom decile is at least 5. This makes our XSMOM strategies available from November, 1991 to May, 2017.

\subsection{Volatility scaling weights}

The core idea of volatility scaling approaches is to control the weight of each instrument to be inversely proportional to its volatility. To adopt the volatility scaling appraches, the first step is to estimate this volatility. Here, we calculate the 6 -month realised volatility using the method of Barroso and 
Table 1: Summarized Statistics.

\begin{tabular}{|c|c|c|c|c|c|c|c|}
\hline No & Contract & Bloomberg Ticker & Sector & Start & End & Mean & $\mathrm{SD}$ \\
\hline 1 & Aluminum & LMAHDS03 Comdty & commodity & Jun-87 & May-17 & 0.022 & 0.245 \\
\hline 2 & Brent & CO1 Comdty & commodity & Jun- 88 & May-17 & 0.121 & 0.416 \\
\hline 3 & Cocoa & CC1 Comdty & commodity & Jan- 86 & May-17 & 0.035 & 0.260 \\
\hline 4 & Coffee & KC1 Comdty & commodity & Jan-86 & May-17 & 0.064 & 0.396 \\
\hline 5 & Copper & LMCADS03 Comdty & commodity & Apr-86 & May-17 & 0.108 & 0.405 \\
\hline 6 & Corn & C 1 Comdty & commodity & Jan-86 & May-17 & 0.058 & 0.276 \\
\hline 7 & Cotton & CT1 Comdty & commodity & Jan-86 & May-17 & 0.047 & 0.303 \\
\hline 8 & Gas Oil & QS1 Comdty & commodity & Jan-86 & May-17 & 0.103 & 0.410 \\
\hline 9 & Gold & GC1 Comdty & commodity & Jan-86 & May-17 & 0.046 & 0.150 \\
\hline 10 & Heating Oil & HO1 Comdty & commodity & Jan-86 & May-17 & 0.112 & 0.397 \\
\hline 11 & Lean Hogs & LH1 Comdty & commodity & Jan- 86 & May-17 & 0.036 & 0.235 \\
\hline 12 & Live Cattle & LC1 Comdty & commodity & Jan-86 & May-17 & 0.032 & 0.111 \\
\hline 13 & Natural Gas & NG1 Comdty & commodity & Apr-90 & May-17 & 0.166 & 0.725 \\
\hline 14 & Nickel & LMNIDS03 Comdty & commodity & Jan- 87 & May-17 & 0.158 & 0.673 \\
\hline 15 & Platinum & JA1 Comdty & commodity & Jan-86 & May-17 & 0.038 & 0.240 \\
\hline 16 & Unleaded & XB1 Comdty & commodity & Oct- 05 & May-17 & 0.086 & 0.452 \\
\hline 17 & Silver & SI1 Comdty & commodity & Jan-86 & May-17 & 0.064 & 0.257 \\
\hline 18 & Soy Meal & SM1 Comdty & commodity & Jan-86 & May-17 & 0.059 & 0.270 \\
\hline 19 & Soy Oil & BO1 Comdty & commodity & Jan-86 & May-17 & 0.055 & 0.251 \\
\hline 20 & Soybeans & S 1 Comdty & commodity & Jan- 86 & May-17 & 0.050 & 0.246 \\
\hline 21 & Sugar & SB1 Comdty & commodity & Jan-86 & May-17 & 0.091 & 0.370 \\
\hline 22 & Wheat & W 1 Comdty & commodity & Jan-86 & May-17 & 0.046 & 0.276 \\
\hline 23 & WTI & CL1 Comdty & commodity & Jan-86 & May-17 & 0.100 & 0.378 \\
\hline 24 & Zinc & LMZSDS03 Comdty & commodity & Jan-89 & May-17 & 0.087 & 0.401 \\
\hline 25 & AUS 3Y & YM1 Comdty & bond & Dec-89 & May-17 & 0.005 & 0.019 \\
\hline 26 & AUS $10 \mathrm{Y}$ & XM1 Comdty & bond & Sep-87 & May-17 & 0.004 & 0.015 \\
\hline 27 & EURO 2Y & DU1 Comdty & bond & Mar-97 & May-17 & 0.005 & 0.013 \\
\hline 28 & EURO $5 \mathrm{Y}$ & OE1 Comdty & bond & Oct-91 & May-17 & 0.016 & 0.041 \\
\hline 29 & EURO 10Y & RX1 Comdty & bond & Nov-90 & May-17 & 0.029 & 0.065 \\
\hline 30 & EURO 30Y & UB1 Comdty & bond & Oct-98 & May-17 & 0.031 & 0.134 \\
\hline 31 & CA $10 \mathrm{Y}$ & CN1 Comdty & bond & Sep-89 & May-17 & 0.016 & 0.071 \\
\hline 32 & JP 10Y & JB1 Comdty & bond & Jan-86 & May-17 & 0.013 & 0.040 \\
\hline 33 & UK $10 \mathrm{Y}$ & G 1 Comdty & bond & Jan-86 & May-17 & 0.007 & 0.086 \\
\hline 34 & US $2 \mathrm{Y}$ & TU1 Comdty & bond & Jun-90 & May-17 & 0.003 & 0.027 \\
\hline 35 & US $5 \mathrm{Y}$ & FV1 Comdty & bond & May-88 & May-17 & 0.009 & 0.052 \\
\hline 36 & US $10 \mathrm{Y}$ & TY1 Comdty & bor & Jan-86 & May-17 & 0.009 & 0.197 \\
\hline 37 & US $30 \mathrm{Y}$ & US1 Comdty & bond & Jan-86 & May-17 & 0.021 & 0.113 \\
\hline 38 & AUD/USD & AD1 Curncy & currency & Jan- 87 & May-17 & 0.008 & 0.129 \\
\hline 39 & $\mathrm{CAD} / \mathrm{USD}$ & CD1 Curncy & currency & Apr-86 & May-17 & 0.005 & 0.089 \\
\hline 40 & EUR/USD & EC1 Curncy & currency & May-98 & May-17 & -0.001 & 0.103 \\
\hline 41 & JPY/USD & JY1 Curncy & currency & May-86 & May-17 & 0.017 & 0.120 \\
\hline 42 & NZD/USD & NV1 Curncy & currency & May-97 & May-17 & 0.018 & 0.133 \\
\hline 43 & NOK/USD & NO1 Curncy & curre & May-02 & May-17 & -0.007 & 0.128 \\
\hline 44 & SEK/USD & SE1 Curncy & currency & May-02 & May-17 & 0.004 & 0.122 \\
\hline 45 & CHF/USD & SF1 Curncy & currency & Apr-86 & May-17 & 0.021 & 0.110 \\
\hline 46 & GBP/USD & BP1 Curncy & currency & May-86 & May-17 & 0.001 & 0.113 \\
\hline 47 & SPI & XP1 Index & index & May-00 & May-17 & 0.052 & 0.174 \\
\hline 48 & $\mathrm{CAC}$ & CF1 Index & index & Jan-90 & May-17 & 0.064 & 0.215 \\
\hline 49 & DAX & GX1 Index & index & Nov-90 & May-17 & 0.112 & 0.235 \\
\hline 50 & FTSE/MIB & ST1 Index & index & Mar-04 & May-17 & -0.013 & 0.211 \\
\hline 51 & TOPIX & TP1 Index & index & May-90 & May-17 & 0.020 & 0.237 \\
\hline 52 & AEX & FXNL Index & index & Jan-90 & May-17 & 0.080 & 0.242 \\
\hline 53 & IBEX & IB1 Index & index & Jul-92 & May-17 & 0.088 & 0.248 \\
\hline 54 & FTSE & Z 1 Index & index & Jan-90 & May-17 & 0.062 & 0.158 \\
\hline 55 & S\&P 500 & SP1 Index & index & Jan-90 & May-17 & 0.091 & 0.169 \\
\hline
\end{tabular}


Santa-Clara (2015), which is an average of squared previous 126 daily returns. The equation is shown as follows:

$$
\sigma_{t}^{2}=\frac{21 \sum_{j=0}^{125} r_{W M L, d_{t-1-j}}^{2}}{126}
$$

where $\sigma_{t}$ denotes the volatility of winner minus loser (WML) series of the XSMOM strategy at time $t$, and $r_{W M L, d}$ is the return of WML series. Given this method is tractable and aligned with the 6 months look-back period, it is used throughout the rest of the paper.

Then, the return of CVS based momentum strategy $r_{W M L, t}^{C V S}$ is given by:

$$
r_{W M L, t}^{C V S}=\frac{\sigma_{\text {target }}}{\sigma_{t}} r_{W M L, t}
$$

where $\sigma_{\text {target }}$ is the target annualised volatility of the portfolio, $\sigma_{t}$ is the realised volatility of 6-month returns calculated from Equation 1. According to both Moskowitz et al. (2012) and Barroso and Santa-Clara (2015), the monthly target volatility for futures investment is reasonably considered as $12 \%$. Hence, we use this figure as the target volatility in our study.

In the DVS approach, the first step is to calculate the conditional expected WML returns, which is estimated depending on the market status and realised volatility. Given that the investors' market expectation is highly related to market conditions, we use the bear market indicator to proxy the market status. Similar to Daniel and Moskowitz (2016), we define the bear market indicator to be equal to 1 if the cumulative returns of the market index in the past 24 months are negative and 0 otherwise. In this study, we employ the MSCI index as the market index because it reflects market status immediately.

Apart from the market status, market expected return is also influenced by the ex-ante realised volatility as high volatility lowers market expectation and vice versa. Hence, we capture how ex-ante realised volatility and bear market indicator impact WML return using the method of Daniel and 
Moskowitz (2016), which is shown as follows:

$$
R_{W M L, t}=\gamma_{0}+\gamma_{B} I_{B, t-1}+\gamma_{\sigma_{m}^{2}} \sigma_{m, t-1}^{2}+\gamma_{i n t} I_{B, t-1} \sigma_{m, t-1}^{2}+\epsilon_{t},
$$

where the dependent variable $R_{W M L, t}$ is the monthly WML return, $I_{B, t-1}$ denotes the lagged bear market indicator, $\sigma_{m, t-1}^{2}$ represents the lagged re-

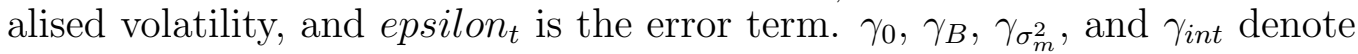
the constant and coefficients for corresponding explanatory variables. Then, the conditional expected WML return over the coming month, $E\left(R_{W M L, t}\right)$, is calculated by the combination of realised volatility and bear market indicator in current month as follow:

$$
E\left(R_{W M L, t}\right)=\gamma_{0, t-1}+\gamma_{i n t, t-1} * I_{B, t-1} * \sigma_{m, t-1}^{2},
$$

where $\gamma_{0, t-1}$ and $\gamma_{i n t, t-1}$ are the estimated coefficients in last period, as the expected WML return of time $t$ is determined by the market status and volatility at time $t-1$.

After the clarification of the relationship between market expected return and realised volatility, we compute the return of DVS based momentum strategy, $r_{W M L, t}^{D V S}$, as:

$$
r_{W M L, t}^{D V S}=\left(\frac{1}{2 \lambda}\right) \frac{E\left(R_{W M L, t+1}\right)}{\sigma_{t}^{2}} r_{W M L, t}
$$

where $r_{W M L, t}$ represents the WML return, $\lambda$ is a time-varying parameter which makes the volatility of DVS based XSMOM returns equals the volatility of weighted average returns of four market indices.

\subsection{Benchmark strategies}

There are two benchmarks used in this study, buy-and-hold and TSMOM strategies. We consider both equally weighted and time-varying volatility weighting schemes to determine the position sizes of the two benchmarks. This returns us four different benchmark strategies. First, in an equally

weighted buy-and-hold strategy, the portfolio return, $r_{t}^{b n h}$, in an asset pool 
consisting of $S$ instruments at time $t$ is given by:

$$
r_{t}^{b n h}=\frac{1}{S_{t}} \sum_{s=1}^{S_{t}} r_{t}^{s}
$$

where $s$ denotes each individual instrument, and $r_{t}^{s}$ is the monthly return of $s$ asset.

Next, in a TSMOM strategy, a positive signal is constructed when the past period return is positive, while a negative signal is generated when the past return is negative. We set the parameters the same as in our XSMOM strategy with a look-back period of 6 months and a holding period of 1 month. Hence, the return of an equally weighted (unscaled) TSMOM strategy at time $t$ is calculated as:

$$
r_{t}^{T S M O M}=\frac{1}{S_{t}} \sum_{s=1}^{S_{t}} \operatorname{sign}\left(r_{t-6, t}^{s}\right) r_{t}^{s},
$$

where the past 6 months return sign, $\operatorname{sign}\left(r_{t-6, t}^{s}\right)$, is either 1 if the 6 months return is positive, and -1 otherwise.

In addition to the above equally weighted benchmarks, we also apply a time-varying volatility adjusted weighting shceme to the buy-and-hold and TSMOM strategies. According to Moskowitz et al. (2012) and Kim et al. (2016), the volatility scaled benchmarks should outperform their original equally weighted version. To implement this approach, we first calculate the time-varying volatility which is an annualised exponentially weighted variance of the past returns. In particular, this estimated variance $\sigma_{t, B}^{2}{ }^{3}$ for each instrument is calculated as follows:

$$
\sigma_{t, B}^{2}=126 \sum_{i=0}^{\infty}(1-\delta) \delta^{i}\left(r_{t-1-i}-\bar{r}_{t}\right)^{2},
$$

where the weights $(1-\delta) \delta^{i}$ add up to one, and $\bar{r}_{t}$ is the exponentially weighted average return computed similarly. The parameter $\delta$ is chosen so that the

\footnotetext{
${ }^{3}$ In order to differentiate this volatility to the $\sigma_{t}^{2}$ estimated in Equation 1, we use subscript $B$ standing for volatility of benchmarks.
} 
center of mass of the weights $\delta /(1-\delta)=60$ days as in Moskowitz et al. (2012).

To measure the scaled returns of buy-and-hold trading strategy, we calculate its position signals in the same way as in Equation 6 but allow the portfolio weight for each instrument to be given as a function of its ex-ante realised volatility. For each asset, we generate the volatility weighted factor to scale the returns. Then, we average all the weighted returns to result the portfolio returns of scaled buy-and-gold strategy. The equation of this approach is exhibited as follow:

$$
r_{t}^{b n h, s c a l e d}=\frac{1}{S_{t}} \sum_{s=1}^{S_{t}} \frac{22.6 \%}{\sigma_{t, B}^{s}} r_{t}^{s},
$$

where $\sigma_{t, B}^{s}$ is the volatility estimated by Equation $8, S_{t}$ are the number of instruments at time $t, r_{t}^{s}$ is the return of asset $s$. According to the method of Moskowitz et al. (2012), the target volatility of $22.6 \%$ is re-estimated so that it is equal to the realised volatility of an equal weighted buy-and-hold portfolio.

Finally, after the combination of scaling factor and Equation 7, the portfolio returns of scaled TSMOM strategy is given by:

$$
r_{t}^{T S M O M, s c a l e d}=\frac{1}{S_{t}} \sum_{s=1}^{S_{t}} \operatorname{sign}\left(r_{t-6, t}^{s}\right) \frac{22.6 \%}{\sigma_{t, B}^{s}} r_{t}^{s},
$$

where $\operatorname{sign}\left(r_{t-6, t}^{s}\right)$ is the signal factor as in Equation 7.

\section{Empirical results}

\subsection{Momentum crashes in futures markets}

As is defined in Daniel and Moskowitz (2016), momentum crash is the period when the cumulative returns of the bottom decile are significantly higher than the cumulative returns of the top decile. This normally occurs 
during financial stress. To motivate our application of volatility scaling approaches, we first identify the momentum crashes in futures markets in this sub-section.

We plot the cumulative returns ${ }^{4}$ of top and bottom deciles in XSMOM strategy from 1991 to 2017 and report it in Figure 1. It can be seen that at the beginning of our XSMOM strategy (1991-1993), the cumulative returns of the bottom decile are slightly higher than the ones in the top decile. This is probably caused by the Soviet Union collapse ${ }^{5}$ and Gulf War $^{6}$. It is a potential momentum crash, but we are not able to identify it as we do not have enough data to calculate the aforementioned 24 months cumulative returns. Then, we focus on the crash resulted from the 2007-2008 financial crisis.

In Figure 1, it can be seen that the cumulative returns of top decile drop significantly from 2007 and become highly volatile between 2007 and 2011, reflecting great uncertainty in the market. Since the bear market indicators for momentum crash are estimated by the 24 months cumulative returns, we investigate the momentum crash between 2009 and 2013. Then, we display the sub-period (2009-2013) cumulative returns in Figure 2 and find that the cumulative returns of bottom decile are higher than the cumulative returns of top decile. Hence, we conclude the observation of the momentum crash caused by the financial crisis in futures markets.

\subsection{Constant versus dynamic volatility scaling approach}

As both CVS and DVS approaches aim to reduce the momentum losses during times of financial stress, we investigate the effect of volatility scaling during 2007-2008. In particular, we separate the entire sample period

\footnotetext{
${ }^{4}$ We assume the investors invest 100 dollars in the strategy, and cumulative returns $(C R)$ is calculated as : $C R=100 * \exp \left(\sum_{i=1}^{n} \ln \left(1+r_{i}\right)\right)$.

${ }^{5}$ Feldstein (1998) claim that the collapse crashes the currencies exchange rates in Europe and triggers Black Wednesday.

${ }^{6}$ Guo et al. (2005) and Kilian (2009) demonstrate that the war significantly impact the supply of crude oil, which further shocks the prices of crude related futures and leads to financial stress.
} 
Figure 1: Cumulative returns of XSMOM top and bottom deciles.

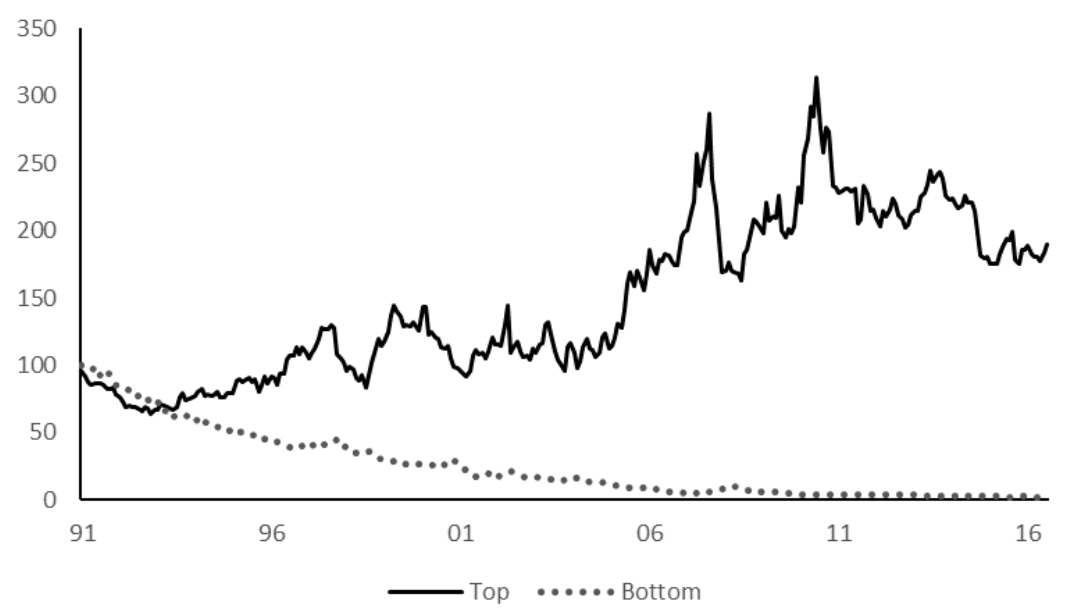

into 3 sub-periods, including pre-crisis, crisis and post-crisis, for a thorough analysis. The first sub-period covers the time between November, 1991 and December, 2006, which is before the beginning of 2007-2008 financial crisis. The second sub-period (crisis period) spans January, 2007 to December, 2010, since the bear market indicators keep equal 1 until the end of 2010 . The last sub-period, i.e. the post-crisis period, is from January, 2011 to May, 2017.

To understand the dynamics of CVS and DVS based XSMOM strategies, we regress their returns on four market indices proxying different asset classes as well as the Fama-French and Carhart three factor models (Fama and French, 1993; Carhart, 1997) representing size, value and momentum effects. The regression equation is shown as:

$r_{t}=\alpha+\beta_{1} m s c i_{t}+\beta_{2} g s c i_{t}+\beta_{3} a g g r_{t}+\beta_{4} d i n x_{t}+\beta_{5} s m b_{t}+\beta_{6} h m l_{t}+\beta_{7} u m d_{t}+\epsilon_{t}$,

where $r_{t}$ denotes the returns of CVS or DVS XSMOM strategies, sci $_{t}$, gsci, $a g g r_{t}, \operatorname{din}_{t}$ are the returns of four market indices introduced in Section 2.1, $s m b_{t}$ (size), $h m l_{t}$ (value) $u m d_{t}$ (momentum) are the three aforementioned risk factors. The results of this regression are shown in Table 2. 
Table 2: Constant versus dynamic volatility scaling approaches.

\begin{tabular}{|c|c|c|c|c|c|c|c|c|c|}
\hline \multicolumn{10}{|c|}{ Panel A Comparison in overall period } \\
\hline Date & strategies & alpha & msci & gsci & $\operatorname{din} x$ & aggr & $\mathrm{smb}$ & $\mathrm{hml}$ & umd \\
\hline \multirow[t]{2}{*}{ 1991-2017 } & CVS & $\begin{array}{c}0.0193^{* * *} \\
(2.75)\end{array}$ & $\begin{array}{l}0.280 \\
(1.62)\end{array}$ & $\begin{array}{c}-0.219^{*} \\
(-1.91)\end{array}$ & $\begin{array}{l}0.208 \\
(0.33)\end{array}$ & $\begin{array}{l}-0.617 \\
(-0.68)\end{array}$ & $\begin{array}{c}0.000351 \\
(0.17)\end{array}$ & $\begin{array}{c}-0.00400^{*} \\
(-1.83)\end{array}$ & $\begin{array}{c}-0.00701^{* * *} \\
(-4.00)\end{array}$ \\
\hline & DVS & $\begin{array}{c}0.0143^{* * *} \\
(2.66)\end{array}$ & $\begin{array}{l}0.197 \\
(1.50)\end{array}$ & $\begin{array}{c}-0.156^{*} \\
(-1.78)\end{array}$ & $\begin{array}{l}0.168 \\
(0.35)\end{array}$ & $\begin{array}{l}-0.437 \\
(-0.63)\end{array}$ & $\begin{array}{c}0.000427 \\
(0.27)\end{array}$ & $\begin{array}{c}-0.00293^{*} \\
(-1.76)\end{array}$ & $\begin{array}{c}-0.00512^{* * * *} \\
(-3.83)\end{array}$ \\
\hline \multicolumn{10}{|c|}{ Panel B Comparison in sub-periods } \\
\hline Date & strategies & alpha & msci & gsci & $\operatorname{din} x$ & aggr & smb & $\mathrm{hml}$ & umd \\
\hline \multirow[t]{2}{*}{ 1991-2006 } & CVS & $\begin{array}{c}0.0256^{* * *} \\
(3.32)\end{array}$ & $\begin{array}{l}0.138 \\
(0.66)\end{array}$ & $\begin{array}{l}0.143 \\
(0.74)\end{array}$ & $\begin{array}{l}0.401 \\
(0.62)\end{array}$ & $\begin{array}{c}-0.0000324 \\
(-0.02)\end{array}$ & $\begin{array}{c}-0.000334 \\
(-0.15)\end{array}$ & $\begin{array}{c}-0.00337 \\
(-1.39)\end{array}$ & $\begin{array}{c}-0.00530^{* * *} \\
(-2.98)\end{array}$ \\
\hline & DVS & $\begin{array}{c}0.0187^{* * * *} \\
(3.26)\end{array}$ & $\begin{array}{l}0.0929 \\
(0.65)\end{array}$ & $\begin{array}{c}-0.347^{* * * *} \\
(-3.77)\end{array}$ & $\begin{array}{l}0.313 \\
(0.65)\end{array}$ & $\begin{array}{l}-0.614 \\
(-0.94)\end{array}$ & $\begin{array}{c}0.0000496 \\
(0.03)\end{array}$ & $\begin{array}{c}-0.00240 \\
(-1.33)\end{array}$ & $\begin{array}{c}-0.00381^{* * * *} \\
(-2.87)\end{array}$ \\
\hline \multirow[t]{2}{*}{$2007-2010$} & CVS & $\begin{array}{c}0.00232 \\
(0.11)\end{array}$ & $\begin{array}{l}0.519 \\
(1.02)\end{array}$ & $\begin{array}{l}-0.135 \\
(-0.43)\end{array}$ & $\begin{array}{l}-1.292 \\
(-0.77)\end{array}$ & $\begin{array}{l}1.689 \\
(0.59)\end{array}$ & $\begin{array}{c}-0.00748 \\
(-0.86)\end{array}$ & $\begin{array}{c}-0.00828 \\
(-1.34)\end{array}$ & $\begin{array}{c}-0.00588 \\
(-1.38)\end{array}$ \\
\hline & DVS & $\begin{array}{c}0.00207 \\
(0.13)\end{array}$ & $\begin{array}{l}0.386 \\
(1.03)\end{array}$ & $\begin{array}{l}-0.112 \\
(-0.48)\end{array}$ & $\begin{array}{l}-0.892 \\
(-0.72)\end{array}$ & $\begin{array}{l}1.194 \\
(0.57)\end{array}$ & $\begin{array}{c}-0.00578 \\
(-0.90)\end{array}$ & $\begin{array}{c}-0.00619 \\
(-1.35)\end{array}$ & $\begin{array}{c}-0.00422 \\
(-1.34)\end{array}$ \\
\hline \multirow[t]{2}{*}{ 2011-2017 } & CVS & $\begin{array}{l}0.0217 \\
(1.20)\end{array}$ & $\begin{array}{l}0.341 \\
(0.59)\end{array}$ & $\begin{array}{l}0.185 \\
(0.47)\end{array}$ & $\begin{array}{l}-1.105 \\
(-0.53)\end{array}$ & $\begin{array}{l}1.373 \\
(0.40)\end{array}$ & $\begin{array}{c}0.0108 \\
(1.37)\end{array}$ & $\begin{array}{r}-0.0127 \\
(-1.43)\end{array}$ & $\begin{array}{c}-0.0224^{* * *} \\
(-3.04)\end{array}$ \\
\hline & DVS & $\begin{array}{c}0.0159 \\
(1.11)\end{array}$ & $\begin{array}{l}0.273 \\
(0.60)\end{array}$ & $\begin{array}{l}0.149 \\
(0.48)\end{array}$ & $\begin{array}{l}-1.002 \\
(-0.60)\end{array}$ & $\begin{array}{l}1.408 \\
(0.52)\end{array}$ & $\begin{array}{c}0.00920 \\
(1.49)\end{array}$ & $\begin{array}{c}-0.00988 \\
(-1.41)\end{array}$ & $\begin{array}{c}-0.0170^{* * *} \\
(-2.93)\end{array}$ \\
\hline \multicolumn{10}{|c|}{ Panel C The differences between two approaches (CVS miuns DVS) } \\
\hline Date & & & & & & & & Diff & P-value \\
\hline $1991-2006$ & & & & & & & & 0.007 & 0.0003 \\
\hline $2007-2010$ & & & & & & & & 0.0003 & 0.960 \\
\hline 2011-2017 & & & & & & & & 0.006 & 0.130 \\
\hline 1991-2017 & & & & & & & & 0.005 & 0.002 \\
\hline
\end{tabular}

The CVS represents constant volatility scaling based XSMOM strategy of Barroso and Santa-Clara (2015). The DVS represents dynamic volatility scaling based XSMOM strategy introduced by Daniel and Moskowitz (2016). The msci, gsci, dinx, aggr, smb, hml, umb are the benchmark factors. Panel A displays the results for the overall period. Panel B displays the results for the three sub-periods. Panel C reports the differences between the alphas of CVS minus DVS approaches, where P-values are estimated based on the F-test as in Kim et al. (2016). 
Figure 2: Cumulative returns during momentum crash.

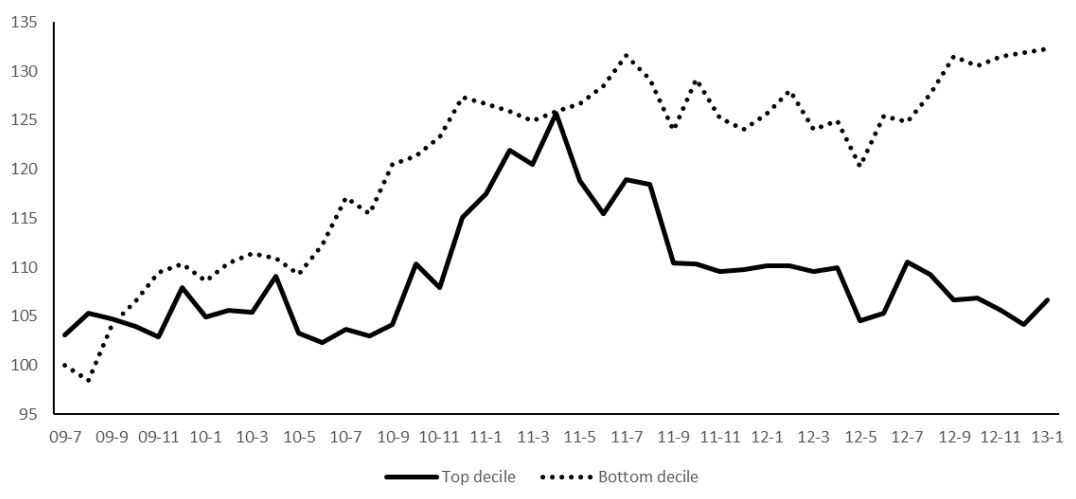

In Table 2 Panel A, we report the regression results of the two scaled XSMOM strategies during the entire sample period. Both approaches show statistically significant alphas at $1 \%$ level. The alpha of the CVS based XSMOM strategy $(1.93 \%)$, is slightly greater than the alpha from the DVS approach $(1.43 \%)$. The returns of both strategies are also strongly related to the umd factors and slightly related to $h \mathrm{ml}$ factors. Moreover, the CVS and DVS based strategies are also negatively related to the gsci factors at $10 \%$ level of significance.

When sub-periods are considered, as shown in Table 2 Panel B, both scaled XSMOM strategies display statistically significant alphas in the precrisis sub-period, but insignificant ones during crisis and post-crisis subperiods. In most of the sample period, the CVS and DVS based returns are strongly correlated with the changes in the momentum factor umd. However, this relationship ceases to hold during the crisis period.

Finally, as is observed in Table 2 Panel C, results show that the CVS based XSMOM strategy outperforms DVS based XSMOM strategy as the difference in alphas is statistically significant at $1 \%$ level (P-value 0.002). However, when different sub-periods are considered, the difference is only statistically significant before the crisis. Specifically, the superiority in alphas is $0.7 \%$ $(\mathrm{p}=0.0003)$ during the pre-crisis period, and then narrows to $0.03 \%(\mathrm{p}=0.960)$ in the financial crisis period. In the post-crisis period, the gap between two 
scaled strategies gets larger at $0.6 \%$ level $(\mathrm{p}=0.130)$ again. To sum $u p$, the CVS is a more efficient scaling method than DVS in the whole period, but the superiority of CVS based XSMOM strategy is almost eliminated during time of financial crisis.

Figure 3 plots the cumulative returns for two 100 dollars investments in CVS and DVS based XSMOM strategies over the period from November, 1991 to May, 2017. Overall, despite the return tendencies of two scaled strategies are similar to each other, the performance of CVS based XSMOM is higher than DVS based XSMOM. More specifically, the cumulative returns of CVS based XSMOM are slightly higher than DVS based XSMOM before 2003, but the superiority expands between 2003 and 2007. Then, the recession during 2007-2008 financial crisis nearly eliminates the difference between the two scaled strategies. After the financial crisis, the recovery of global economic condition improves performance of both scaled strategies with the gap being quite small. These findings are consistent with our prior regression results, where 2007-2008 financial crisis decreases the performance of both scaled XSMOM strategies and almost eliminates the superiority of CVS based XSMOM strategy.

Figure 3: Cumulative returns of CVS and DVS based XSMOM strategies.

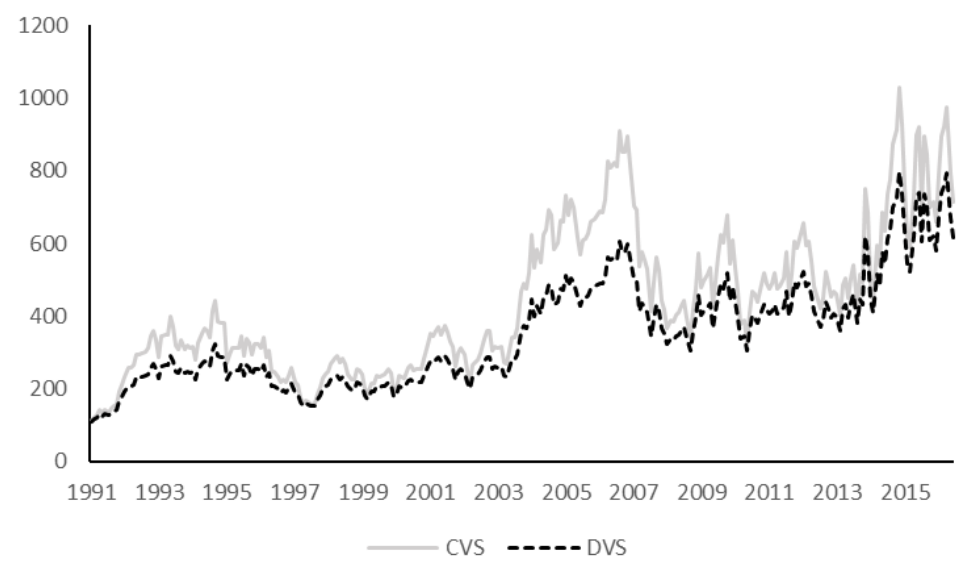




\subsection{Cross-strategy comparison}

In this sub-section, we conduct an extended cross-strategy comparison where the equally weighted buy-and-hold, TSMOM (Moskowitz et al., 2012) and XSMOM as well as the scaled buy-and-hold and TSMOM strategies are included. These benchmarks are added as they are also linked to the volatility scaling approach studied in this paper, and hence, providing valuable comparison. This allows us to evaluate whether the CVS/DVS based XSMOM outperform the benchmarks, resulting in a more robust conclusion. To implement the comparison, we still employ the regression as in Equation 11 to understand the dynamics of all involved strategies. As in the previous sub-section, we also include the trading strategy analysis and cumulative returns plot.

We report the regression results in Table 3. As seen in panel A, the buyand-hold returns are highly related to the four market indices, namely msci, gsci, dinx, and aggr. The only difference is that the scaled buy-and-hold generates an statistically significant alpha while the unscaled buy-and-hold does not. In contrast, the TSMOM returns only have significant coefficients with the msci and umd as shown in Panel B. Both scaled and unscaled TSMOM strategies exhibit alphas at $0.217 \%$ and $0.332 \%$, respectively, which are at $5 \%$ level of significance. Panel $\mathrm{C}$ provides a comparison among the three XSMOM strategies: unscaled XSMOM, CVS and DVS based XSMOM. It can be seen from the results that the volatility scaled strategies have less exposure to the market indices than the original XSMOM strategy. The CVS based XSMOM still generates the highest alpha among the three approaches. Finally, in order to assess whether the alphas of scaled XSMOM strategies are significantly higher than scaled benchmark strategies, we compare the alphas of scaled XSMOM strategies with the other scaled strategies in panel D. According to the negative differences and low p-values, we suggest that both scaled XSMOM strategies significantly outperform the benchmark strategies. 
Table 3: Cross-strategy comparison.

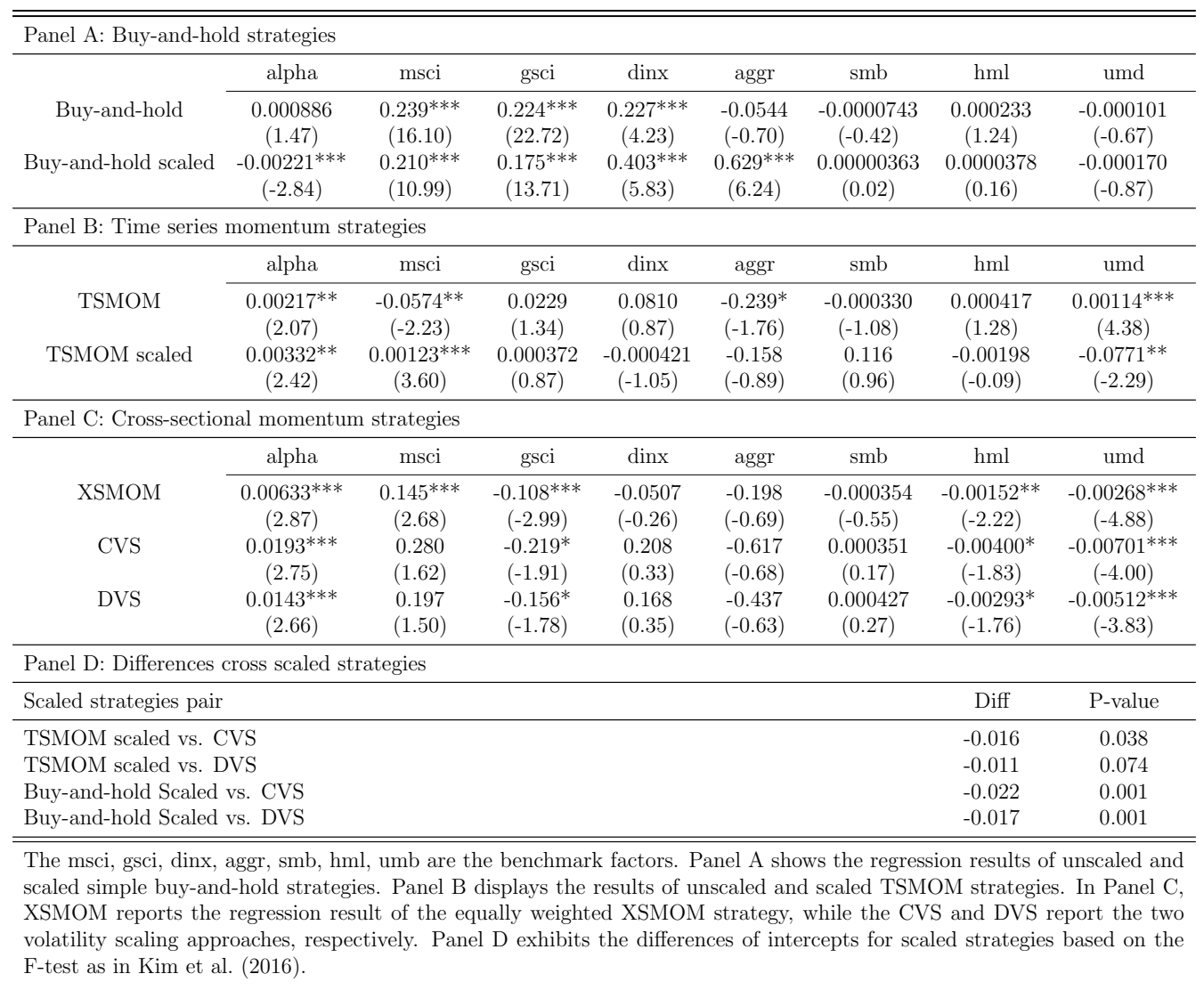


In addition to the regression results, we also plot the expanded cumulative return comparison in Figure 4, where the cumulative returns of both CVS and DVS based XSMOM perform better than the scaled benchmark strategies. These results are consistent with the aforementioned findings in Table 3, where the two scaled XSMOM strategies perform significantly better than any benchmarks. However, it is also witnessed that the strategies based on CVS and DVS approaches exhibit higher volatility than the two benchmarks, indicating lower return-to-risk ratios.

Figure 4: Cumulative returns of all scaled strategies.

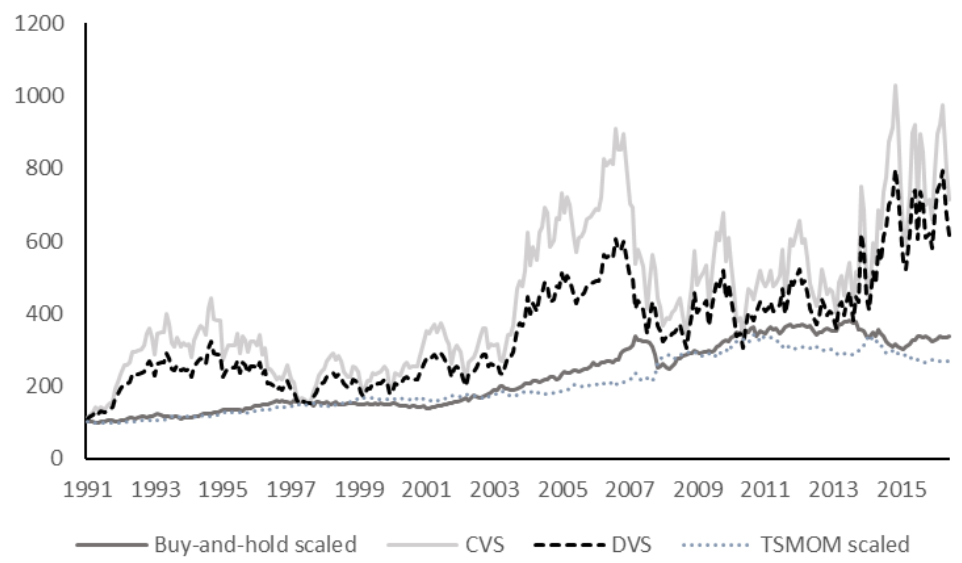

For a thorough investigation of these trading strategies, we further evaluate their performance as summarised in Table 4. First, all the volatility scaled strategies exhibit higher average returns and volatility than the corresponding unscaled strategies. This result is the same as is suggested in Kim et al. (2016). Second, the two scaled XSMOM strategies display the highest average returns of $15.3 \%(\mathrm{CVS})$ and $11.5 \%(\mathrm{DVS})$ per annum, which are twice to three times greater than those of the benchmark strategies. Whereas the Sharp ratios of the two scaled XSMOM strategies are almost equal to each other, but smaller than some of the benchmarks. This suggests that the CVS and DVS approaches lead to greater profitability than their rivals, and at the same time display higher uncertainty. 
Table 4: Performance of CVS and DVS based XSMOM compared to benchmarks.

\begin{tabular}{cccccccc}
\hline \hline Strategies & Average & Volatility & Sharpe & Max & Min & Cumulative & Drawdown \\
\hline buy-and-hold & 0.045 & 0.077 & 0.580 & 0.078 & -0.134 & 1.915 & 0.301 \\
buy-and-hold scaled & 0.051 & 0.084 & 0.605 & 0.074 & -0.131 & 2.361 & 0.270 \\
TSMOM & 0.023 & 0.059 & 0.396 & 0.136 & -0.043 & 0.738 & 0.188 \\
TSMOM scaled & 0.042 & 0.076 & 0.548 & 0.154 & -0.057 & 1.693 & 0.224 \\
XSMOM & 0.048 & 0.123 & 0.393 & 0.101 & -0.099 & 1.825 & 0.297 \\
CVS & 0.153 & 0.391 & 0.392 & 0.515 & -0.287 & 6.261 & 0.645 \\
DVS & 0.115 & 0.297 & 0.386 & 0.439 & -0.224 & 5.164 & 0.532 \\
\hline \hline
\end{tabular}

Each row concludes the annualised summary of covered strategies. In particular, the Average column reports the average annualise returns of each strategy, Volatility column displays the annualised volatility of each strategy, the Sharpe column exhibits the Sharpe ratios of strategies, Max and Min columns report the highest and lowest monthly returns in each strategy; Drawdown shows the peak-to-trough decline periods. All figures are calculated by the monthly returns of each strategies.

\section{Conclusion}

We observe a momentum crash in futures markets during 2009-2013, which is also witnessed in the US stock markets by Daniel and Moskowitz (2016). Then, we compare the performance of two volatility scaling approaches, CVS and DVS, in XSMOM strategies. The regression results suggest that CVS of Barroso and Santa-Clara (2015) produces statistically superior alphas than the DVS of Daniel and Moskowitz (2016) in most of the sample periods. However, the positive difference gets smaller during the crisis period. Furthermore, the CVS based XSMOM strategy exhibits the best profitability among all strategies in a cross-strategy comparison including the equally weighted buy-and-hold, TSMOM and XSMOM as well as scaled buyand-hold and TSMOM strategies. Therefore, we conclude that the CSV is a more efficient volatility scaling method for momentum strategies in futures markets. 


\section{References}

Asness, C. S., Liew, J. M. and Stevens, R. L. (1997), 'Parallels between the cross-sectional predictability of stock and country returns', The Journal of Portfolio Management 23(3), 79-87.

Asness, C. S., Moskowitz, T. J. and Pedersen, L. H. (2013), 'Value and momentum everywhere', The Journal of Finance 68(3), 929-985.

Barroso, P. and Santa-Clara, P. (2015), 'Momentum has its moments', Journal of Financial Economics 116(1), 111-120.

Boguth, O., Carlson, M., Fisher, A. and Simutin, M. (2011), 'Conditional risk and performance evaluation: Volatility timing, overconditioning, and new estimates of momentum alphas', Journal of Financial Economics 102(2), 363-389.

Carhart, M. M. (1997), 'On persistence in mutual fund performance', The Journal of Finance 52(1), 57-82.

Daniel, K. and Moskowitz, T. J. (2016), 'Momentum crashes', Journal of Financial Economics 122(2), 221-247.

Fama, E. F. and French, K. R. (1993), 'Common risk factors in the returns on stocks and bonds', Journal of Financial Economics 33(1), 3-56.

Fama, E. F. and French, K. R. (1998), 'Value versus growth: The international evidence', The Journal of Finance 53(6), 1975-1999.

Feldstein, M. (1998), 'Refocusing the imf', Foreign Affairs-New York $77,20-33$.

Guo, H., Kliesen, K. L. et al. (2005), 'Oil price volatility and us macroeconomic activity', Review-Federal Reserve Bank of Saint Louis 87(6), 669 .

Jegadeesh, N. and Titman, S. (1993), 'Returns to buying winners and selling losers: Implications for stock market efficiency', The Journal of Finance 48(1), 65-91. 
Kilian, L. (2009), 'Not all oil price shocks are alike: Disentangling demand and supply shocks in the crude oil market', The American Economic Review 99(3), 1053-1069.

Kim, A. Y., Tse, Y. and Wald, J. K. (2016), 'Time series momentum and volatility scaling', Journal of Financial Markets 30, 103-124.

Lewellen, J. (2002), 'Momentum and autocorrelation in stock returns', Review of Financial Studies 15(2), 533-564.

Miffre, J. and Rallis, G. (2007), 'Momentum strategies in commodity futures markets', Journal of Banking \& Finance 31(6), 1863-1886.

Moskowitz, T. J. and Grinblatt, M. (1999), 'Do industries explain momentum?', The Journal of Finance 54(4), 1249-1290.

Moskowitz, T. J., Ooi, Y. H. and Pedersen, L. H. (2012), 'Time series momentum', Journal of Financial Economics 104(2), 228-250.

Rouwenhorst, K. G. (1999), 'Local return factors and turnover in emerging stock markets', The Journal of Finance 54(4), 1439-1464.

Shen, Q., Szakmary, A. C. and Sharma, S. C. (2007), 'An examination of momentum strategies in commodity futures markets', Journal of Futures Markets 27(3), 227-256.

Teplova, T. and Mikova, E. (2015), 'New evidence on determinants of price momentum in the japanese stock market', Research in International Business and Finance 34, 84-109.

Wang, K. Q. and Xu, J. (2015), 'Market volatility and momentum', Journal of Empirical Finance 30, 79-91.

Zaremba, A. and Szyszka, A. (2016), 'Is there momentum in equity anomalies? evidence from the polish emerging market', Research in International Business and Finance 38, 546-564. 